\title{
Surveillance, epidemiology and behavioural research to guide HIV prevention policy
}

\section{Andrew Grulich ${ }^{\mathrm{A}}$, Darryl O'Donnell ${ }^{\mathrm{B}}$ and John B. F. de Wit ${ }^{\mathrm{C}}$}

${ }^{\mathrm{A}}$ National Centre in HIV Epidemiology and Clinical Research, The University of New South Wales

${ }^{\mathrm{B}}$ AIDS/Infectious Diseases Branch, NSW Department of Health

${ }^{\mathrm{C}}$ National Centre in HIV Social Research, The University

of New South Wales

Since 2000, HIV prevention in NSW has been overseen by a Health Promotion Sub-Committee of the Ministerial Advisory Committee on HIV and Sexually Transmissible Infections. The Sub-Committee meets quarterly, and its ongoing work demonstrates how reflexive interactions between researchers, community-based and public-sector educators and policy makers can enable the development and implementation of effective evidence-based policy. This facility is highlighted by the following two initiatives led by the Committee.

\section{2: Is HIV on the rise in NSW?}

In 2002, after approximately 15 years of declining HIV rates in NSW, the annual number of new HIV diagnoses increased in NSW; at the same time it also increased in some other Australian states and internationally. Consequently in 2003, the Sub-Committee convened a forum to examine HIV notifications surveillance with the aim of reaching a consensus among researchers, community organisations, health promotion workers, clinicians and policy makers about the meaning and implications of this increase. Disease surveillance officers, as well as social, behavioural and epidemiological scientists were asked to review their data, and to present their findings at the forum. The findings were clear: that HIV diagnoses had increased in NSW, and that the most likely explanation was a true increase in incidence in 2002 related to increasing rates of unprotected anal intercourse. These findings led to increased investment and more focused community-education campaigns alerting the gay community that HIV rates were increasing.

\section{7: Why are HIV notifications in NSW flat?}

By 2007, HIV diagnoses had been increasing in homosexual men for almost a decade in many developed countries. Rates had doubled since the nadir in the late 1990s in some Australian jurisdictions. In contrast, apart from the brief increase in 2002, HIV diagnoses in homosexual men in NSW had remained stable. There was discussion about whether this exceptional pattern was real, or whether diagnoses were increasing and the surveillance system in NSW had not recorded this. In response the Sub-Committee decided that a comprehensive review of the HIV epidemic in NSW was required. This took the form of a Think Tank entitled Why are HIV notifications in NSW flat? ${ }^{1}$ Scientists from across the spectrum of HIV public health were assembled and undertook a six month process of enquiry and review to ensure that all possible explanations for the lack of increase in HIV diagnoses in NSW were considered. The Think Tank assembled scientists, the leadership of community-based organisations, clinicians, public-sector health promoters and those responsible for the Government's policy response to consider the evidence. The result was clear: sustained and sufficient investment in NSW had provided the capacity for health promotion efforts to decrease HIV risk behaviour, which meant that NSW had avoided the increases in HIV rates seen in almost every other epidemic in homosexual men where long-term data were available.

These results, published as a series of 17 articles in the peer-reviewed journal Sexual Health, establish the public health business case for both continued investment in HIV prevention in NSW and the reinvestment in HIV prevention in some other Australian jurisdictions. The editorial for the issue which summarised the findings, was entitled Investment in HIV prevention works: a natural experiment, and concluded that between 1999 and 2006 over 1500 cases of HIV had been prevented by sustained investment in HIV prevention in NSW, at a cost per case prevented of around $\$ 30000 .^{2}$

\section{References}

1. NSW Health. A Think Tank: why are HIV notifications flat in NSW 1998-2006? Consensus statement. 30 April 2007. Available at: http://www.health.nsw.gov.au/ resources/publichealth/sexualhealth/pdf/consensus_ statement.pdf (Cited 22 March 2010).

2. Fairley CK, Grulich AE, Imrie JC, Pitts M. Investment in HIV prevention works: a natural experiment. Sex Health 2008; 5: 207-10. doi:10.1071/SH08017 